$\mathbb{P}$ periodica polytechnica

Chemical Engineering

$52 / 1(2008) 346$

doi: 10.3311/pp.ch.2008-1.01

web: http://www.pp.bme.hu/ch

(c) Periodica Polytechnica 2008

RESEARCH ARTICLE

\section{Electric field controlled flow behaviour of electrorheological fluid}

\author{
Árpád Forberger / Genovéva Filipcsei / Gábor Stépán / Miklós Zrínyi
}

Received 2005-10-13

\begin{abstract}
The main purpose of the present study is to demonstrate the electric field controlled flow behaviour of electrorheological (ER) fluid. We have investigated the relation between the electric field and the change in the viscosity of electrorheological fluids. Different kinds of suspensions are used to search for the proper combination ER fluid for applying in a motor mount. A rotational viscometer is used to get how the viscosity depends on temperature and a special device to measure the effect of electric fields up to $1.8 \mathrm{kVmm}^{-1}$.
\end{abstract}

\section{Keywords}

electrorheology · dielectrophoresis · polymer dispersions . viscosity

\section{Acknowledgement}

This research was supported by the Intel KKK (GVOP-3.2.22004-07-0006/3.0) and NKFP-3A/081/04.

\section{Árpád Forberger}

Department of Physical Chemistry and Materials Science, BME, H-1521, Budapest, Hungary

\section{Genovéva Filipcsei}

Materials Structure and Modelling Research Group of the Hungarian Academy of Sciences, MTA-BME Laboratory of Soft Matters, H-1521, Budapest, Hungary

\section{Gábor Stépán}

Department of Applied Mechanics, BME, H-1521, Budapest, Hungary

\section{Miklós Zrínyi}

Department of Physical Chemistry and Materials Science, BME, H-1521, Budapest, Hungary

\section{Introduction}

Rheological properties of materials are related to their response to applied stress. The field induced chain formation of a large number of organic and inorganic substances such as starch, aluminium oxide, ferric oxide, carbon black, ionexchange resins, and polymer powders have been studied mainly in insulating oils.

Electrostatic spray painting is widely used in order to enhance the paint transfer efficiency. It is based on strong Coulombic interactions between paint particles and surface to be coated. An electrostatic field is created and as a result, the paint particles pick up electrons and become ionised. The grounded work piece attracts the negatively charged paint particles and thus the transfer efficiency is increased significantly. Another electrostatic effect is based on the polarisation of dispersed particles in electric field. Small particles in the size range from about one-micron up to one millimetre dispersed in non-conducting medium, mainly in organic solvents, can respond to an applied electric field by rapidly changing their apparent viscosity. Such a colloidal solution is called electrorheological fluid and the interaction with the field is often designated as dielectrophoresis. The dielectrophoresis refers to the force exerted on the induced dipole moment of an uncharged particle by nonuniform electric field. The polarised particles interact not only with the imposed field, but also with each other. At close spacing these interaction forces can be quite strong. The particles attract each other when aligned parallel to the applied field, or repel each other when in perpendicular alignment. The attractive forces between particles lead to chain formation. The pearl chain structuring makes possible far implication in particulate science. The field induced chain formations of a large number of organic and inorganic substances such as starch, aluminium oxide, ferric oxide, carbon black, ion-exchange resins, and polymer powders have been studied mainly in insulating oils.

Much less is studied and understood the electrorheological effect of pigments in polymer dispersions. Titanium dioxide is one of the most frequently used surface coatings due to its highquality and ecologically acceptable properties. In the present paper the authors first review the basic principle of electrorheol- 
ogy, this is followed by experimental studies on structuring and rheological properties.

\section{Electrorheological effects}

The terms of electrophoresis and dielectrophoresis imply the interplay between electrical phenomena and motion. Dielectrophoresis is the translational motion of neutral particles in nonuniform electric field due to polarisation. Although polarisation effects are often considered to be weak, there are several examples where they are far more effective than Coulomb forces, since polarisation forces can act directly on an uncharged particle. Electrorheology is based on dielectrophoretic effects.

All materials experience forces or torques when subjected to electric field. These interactions are strong in the case of certain solid materials, but rather weak in fluid systems. In order to enhance the influence of the external fields on the fluid-like properties, it is necessary to combine solid like and fluid like behaviours. Since polymer dispersions contain substantial amount of liquid, it is possible to fabricate a field sensitive fluid, the socalled complex fluid. A complex fluid contains dispersed small particles in the size range of nanometres to micrometers [1]. Responding to an applied field the particles in the fluid couple the viscosity, yield stress and other physical properties to the exterior. The response time can approach the order of millisecond [2]. If we incorporate polarisable colloidal particles into a polymer solution, due to an imposed electric field, the particles experience a dielectrophoretic force. Since the particles move together with the fluid all of the forces acting on the particles are transmitted directly to the polymer dispersion resulting in peculiar rheological behaviour.

If an electrorheological fluid is exposed to an external field, two distinct types of interaction can be identified: field-particle interaction, as well as particle-particle interaction. If the field is nonuniform, then the field-particle interactions are dominant. Particles experience a dielectrophoretic force, $f_{D E P}$. As a result, the particles are attracted to regions of stronger field intensities [3]:

$$
f_{D E P}=2 \pi \varepsilon_{1} R^{3} K \nabla E^{2}
$$

where $\varepsilon_{1}$ denotes the dielectric permittivity of the solvent, $R$ stands for the radius of colloidal particle. The $\nabla$ symbol represents the gradient operator. The quantity $K$ is known as the Clausius-Mossotti function that provides a measure of the strength of the effective polarisation of the spherical particle with a dielectric permittivity of $\varepsilon_{2}$ :

$$
K\left(\varepsilon_{1}, \varepsilon_{2}\right)=\frac{\varepsilon_{2}-\varepsilon_{1}}{\varepsilon_{2}+2 \varepsilon_{1}}
$$

$E q$. 1 reveals the most important feature of electrophoretic effects. It says that the electrophoretic force is proportional to the particle volume of the individual particles dispersed in the liquid. $f_{D E P}$ is also proportional to the dielectric permittivity of the medium in which the colloidal particles are suspended. It is important to mention that the dielectrophoretic force vector is directed along the gradient of the square of electric field intensity, which in general, is not parallel to the electric field vector E. It is also seen that $f_{D E P}$ depends on the sign and magnitude of the Clausius-Mossotti function. In case of $K>0$ the colloidal particles are attracted to electric field intensity maxima and repelled from minima. The field direction does not play any role.

In uniform fields the situation is completely different. Due to the lack of field gradient, there are no attractive or repulsive field-particle interactions. The particle-particle interaction becomes dominant. The imposed field induces electric dipoles. As a result, mutual particle interactions occur if the particles are so closely spaced that the local field can influence their neighbours. This mutual interaction can be very strong leading to significant change in the structure of particle ensembles. The particles attract each other when aligned end to end, and repel each other in side by side situation. Due to the attractive forces pearl chain structure may develop. This field-induced chaining has been found to influence the value of viscosity and yield stress. In nonuniform fields both phenomena, the dielectrophoretic force as well as the structure formation occurs.

Electrorheological fluids are suspensions of polarisable solid particles in a fluid medium of low electrical conductivity. An electric field is applied to increase the resistance to flow and with the field it is totally controllable. Without the control field they readopt their original rheological properties, such low viscosity. These fluids can be used as fast reacting interfaces between control electronics and mechanical system in actuators, clutches, vibration dampers, motor mounts and valves in hydraulic systems as well. There are several experiments available that discuss the rheological behaviour, properties and adaptability in active control applications of ER fluids. Still there are only a few commercial applications. One is an MR damper developed by GM. Mostly automotive adaptations are the research priorities, in most cases reducing vibration, substituting moving-rotating parts with ER fluids. In the late 1980s GM carried out a study on the application potential of ER fluids, and concluded that a major hurdle was the low yield stress of the ER fluids. Several benefits are known, so let us discuss some. First, after switching off the electric field the fluid shifts very fast, within a few milliseconds, which is essential in active controls. And this process is totally reversible. Today dampers in motor mounts are used always as passive suspension systems, so one cannot change their properties (for example stiffness and damping). And here comes the benefits of ER fluids with their controllable viscosity and damping ratio.

\section{Experiments}

\subsection{Preparation of the ER fluid}

The model system of our studies was based on the dispersion of electric field sensitive powder mixture containing mostly $\mathrm{Ba}$, $\mathrm{La}, \mathrm{Y}, \mathrm{Ce}$ in silicon oil. The average particle size is $60 \mu \mathrm{m}$. The silicon oil (DC200) was obtained from Fluka. The viscosity of 
the silicon oil was 1 and 2 Pas at $25{ }^{0} \mathrm{C}$. The concentration of the ER suspension was varied in a wide range from 30 up to 50 $\mathrm{w} \%$.

\subsection{Experimental Methods}

In order to investigate the effect of the electric field on the viscosity of ER fluid a home-made apparatus was developed as shown in Fig. 1 Printed Board Circuit were used for the elec-

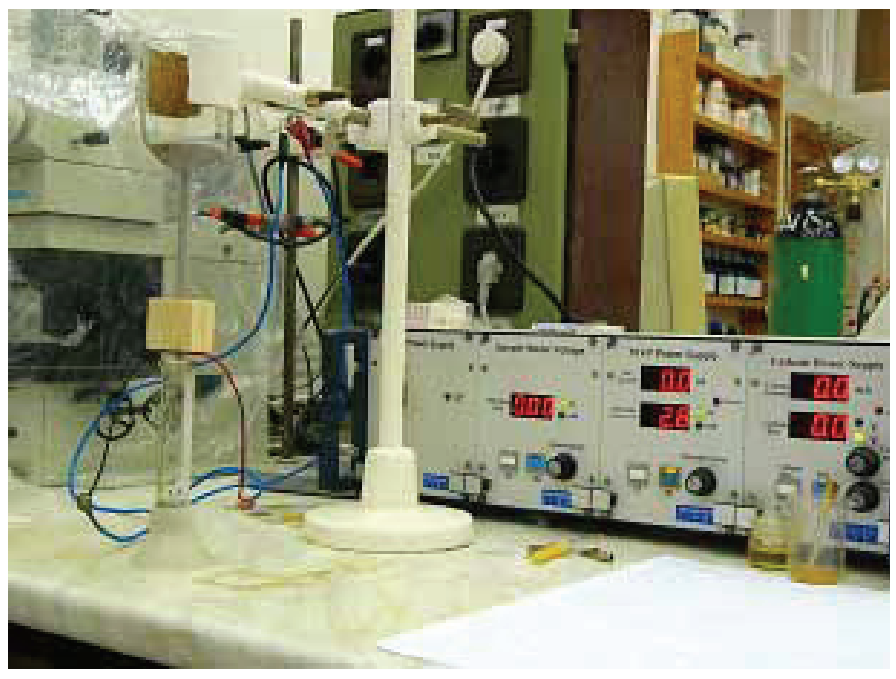

Fig. 1. Home-made apparatus to determine the viscosity in DC electric field

trodes. The electrode distance was varied from $3 \mathrm{~mm}$ up to 7 $\mathrm{mm}$. The flow time of $10 \mathrm{ml}$ ER fluid through the glass capillary was measured at different electric field intensities. All measurements were performed at 20 and $50{ }^{\circ} \mathrm{C}$. The specific viscosity ( $\eta_{\text {spec }}$ ) of the ER fluid at certain voltages (electric fields) can be calculated according to the Haigen-Poiseuille law:

$$
\eta_{\text {spec }}=\frac{t-t_{0}}{t_{0}} \approx \frac{\eta-\eta_{0}}{\eta_{0}}
$$

where $t$ is the flow time at a certain field intensity, $t_{0}$ is the flow time without electric field, $\eta_{0}$ is the viscosity of the ER fluid, $\eta$ is the viscosity in electric field.

The viscosity of the ER fluid without external field was measured by HAAKE Rheostress RS100 rheometer at various temperatures from 20 up to $50{ }^{0} \mathrm{C}$.

\section{Results and Discussion}

\subsection{Dynamic Viscosity of the ER Fluid}

In order to investigate the effect of the DC electric field on the dynamic viscosity of the ER fluid it is essential to determine its dynamic viscosity at different temperatures. Fig. 2 shows the temperature dependence of the dynamic viscosity of the ER fluid. The concentration of the ER fluid was varied from 30 up to $50 \mathrm{w} \%$. The dynamic viscosity of the ER fluid decreases with increasing the temperature, as expected. The dynamic viscosity at $20{ }^{0} \mathrm{C}$ and $50{ }^{0} \mathrm{C}$ was used for the measurement in electric field as $\eta_{0}$.

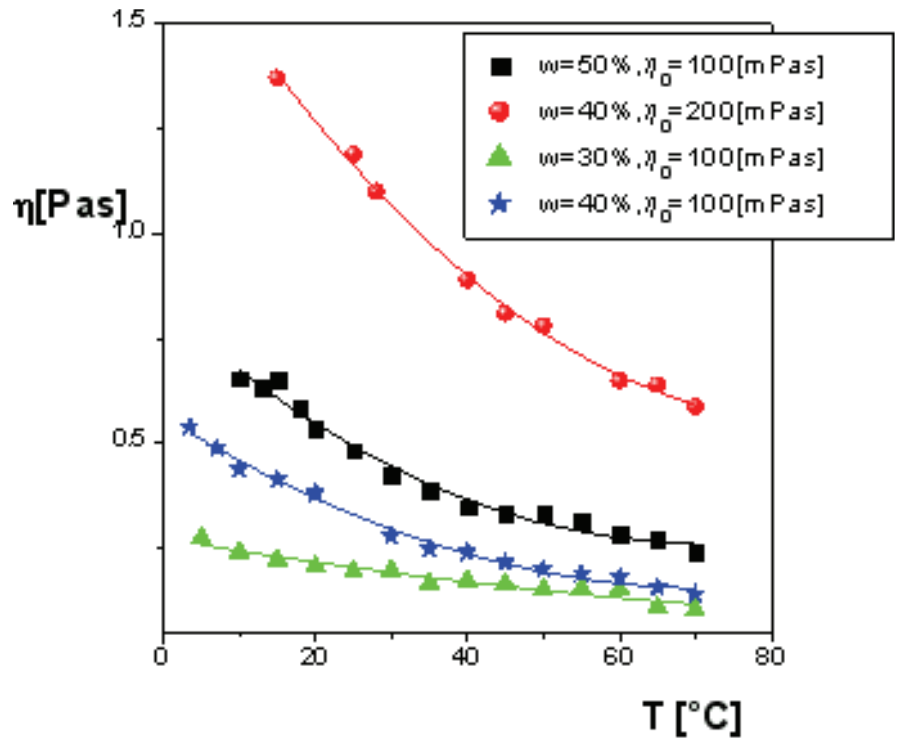

Fig. 2. The effect of the temperature on the dynamic viscosity of the ER fluid

\subsection{Effect of the Electric Field on the Dynamic Viscosity of ER Fluid}

To investigate the effect of the electric field on the dynamic viscosity the flow time of $10 \mathrm{ml}$ ER fluid was measured at different field intensities. Each measurement was continued until the flow of the ER fluid was stopped at a certain field intensity. Using the flow time, the specific viscosity was calculated according to Eq. (1).

Fig. 3 shows the effect of the electric field on the specific viscosity of ER fluid at 20 and $50{ }^{\circ} \mathrm{C}$. The concentration of the ER fluid was $40 \mathrm{w} \%$. The electrode distance was $3 \mathrm{~mm}$. By increas-

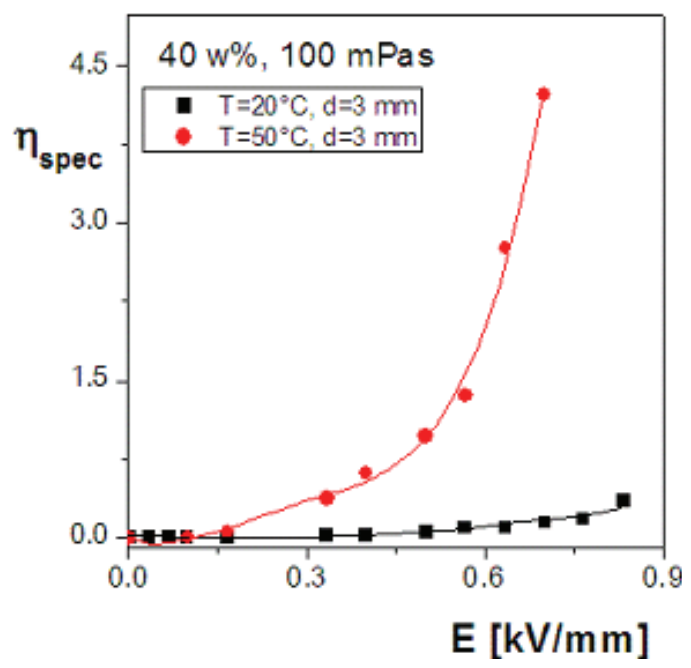

Fig. 3. The effect of the temperature and electric field on the specific viscosity of ER fluid

ing the electric field intensity, the specific viscosity of the ER fluid increases significantly as seen in the Fig. 3. At $20{ }^{0} \mathrm{C}$ the flow of the ER fluid was stopped at $0.833 \mathrm{kV} / \mathrm{mm}$ field intensity. At $50{ }^{0} \mathrm{C}$ the field intensity that was needed to stop the flow was $0.7 \mathrm{kV} / \mathrm{mm}$. As increasing the temperature causes the viscosity of the ER fluid to decrease, this could be the explanation of the smaller field intensity that is needed to stop the flow. 
In uniform field, because of the lack of field gradient, there are no attractive or repulsive field-particle interactions; therefore particle-particle interactions become dominant. The imposed field induces electric dipoles. If the particles are spaced closely enough, for their field to reach their neighbours, mutual particle interactions occur. These interactions can be very strong leading to significant change in the structure of the particle ensemble. The particles attract each other when aligned end to end, and repel each other when placed side by side. Due to the attractive forces pearl chain structure develops (Fig. 4). As a result the viscosity of the suspension increases dramatically and when the field intensity exceeds a critical value, the ER fluid turns into a solid.

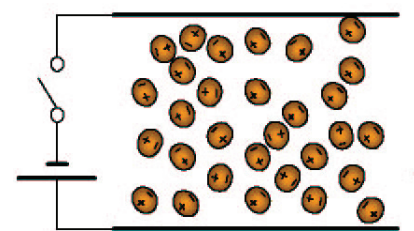

a

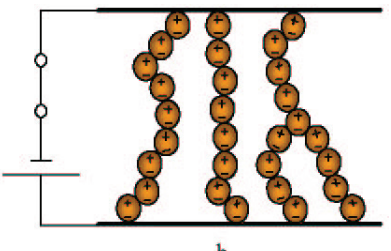

Fig. 4. Schematic representation of the electrorheological effect a) without electric field, b) in uniform electric field

For the technical application it is important to investigate the effect of the ER fluid concentration on the flow behaviour. Fig. 5 shows the specific viscosity of the ER fluid with 3 different concentrations versus the electric field intensity at $20{ }^{\circ} \mathrm{C}$ and $3 \mathrm{~mm}$ electrode distance. If the concentration of the ER fluid is $30 \mathrm{w} \%$,

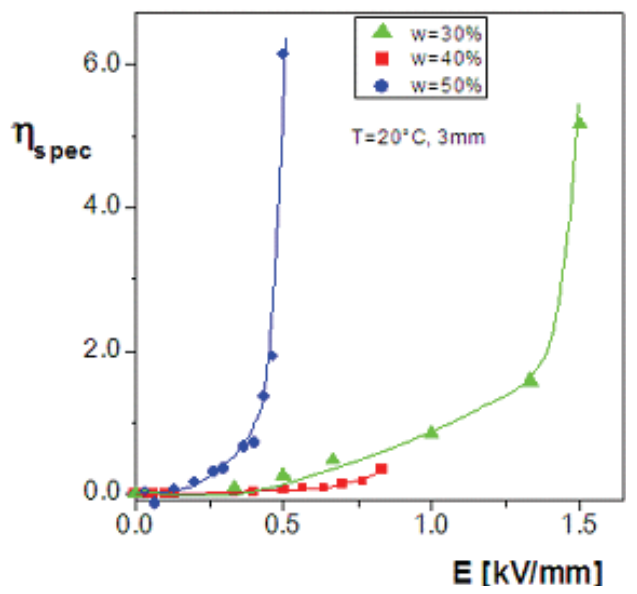

Fig. 5. Effect of the electric field intensity on the specific viscosity of the ER fluid with different concentrations

the particle alignments due to the electric field are not strong enough to stop the flow of the fluid at low electric field intensity. $1.5 \mathrm{kV} / \mathrm{mm}$ field intensity is required to stop the flow. If the concentration of the ER fluid is high, $50 \mathrm{w} \%$, the particle chains are strong enough to stop the flow at smaller field intensity $(0.5 \mathrm{kV} / \mathrm{mm})$. However, the sedimentation of the particles is notable due to particle aggregation and handling of the fluid is quite difficult because of the high viscosity. In the intermediate case, $0.833 \mathrm{kV} / \mathrm{mm}$ electric field intensity is necessary to stop the flow and the viscosity of the ER fluid is convenient for its application.
It has to be mentioned that in spite of the high electric field intensity the current uptake is a few $\mu A$. For that reason the power consumption is only a few $m W$. This consumption is negligible for the further possible applications.

In Fig. 6 the effect of the electric field on the specific viscosity of the $30 \mathrm{w} \%$ ER fluid at different electrode distances can be seen. Each measurement was performed at $50^{\circ} \mathrm{C}$. The electrode distance was varied from $3 \mathrm{~mm}$ up to $7 \mathrm{~mm}$.

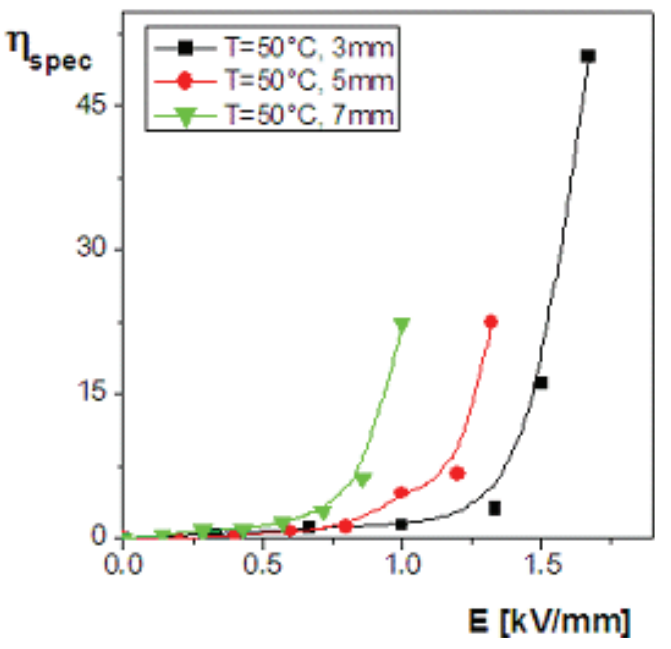

Fig. 6. The effect of the electric field on the specific viscosity of ER fluid at different electrode distances. The temperature and the electrode distances are indicated.

We have found that the distance of the electrodes influences the ER behaviour of our fluid significantly. The larger the gap distance, the weaker is the ER effect. To understand this effect better, further studies are needed.

\section{Conclusion}

We have investigated the effect of the electric field on the specific viscosity of ER fluid. Due to the particle polarization in a uniform electric field, the particles form chain-like structures modifying the viscosity and the flow properties of the ER fluid in a wide range. The effects of the temperature and the electrode distance as well as the concentration of the ER fluid were studied. We can conclude that increasing the temperature and the electrode distance decreases the intensity of the electric field that is needed to stop the flow of the fluid. The concentration for the technical application was found to be $40 \mathrm{w} \%$.

Application of the ER fluids in engineering promises new materials and technologies with a broad spectrum of mechanical applications including micro machines, energy-transducing devices, sensors, bearings and vibration absorbers.

\section{References}

1 Jones TB, Electromechanics of Particles, Cambridge University Press, 1995.

2 Nakano M, Koyama K, Electrorheological Fluids, Magnetorheological Suspensions and their Applications, World Scientific Publishing Co. Pte. Ltd., 1997.

3 Thao R, Roy GD, Electrorheological fluids, World Scientific Publishing Co. Pte. Ltd., 1994. 